\title{
A Comparative Study of the Effects of Platelet-Rich Fibrin, Concentrated Growth Factor and Platelet- Poor Plasma on the Healing of Tooth Extraction Sockets in Rabbits
}

\section{Siying Li}

Southwest Medical University

\section{Hongyi Yang}

Southwest Medical University

\section{Qinyu Duan}

Southwest Medical University

Hongyu Bao

Southwest Medical University

Aodi Li

Southwest Medical University

Wei Li

Southwest Medical University

Yun He ( $\nabla$ heyundaidai@163.com )

Southwest Medical University

\section{Research Article}

Keywords: tooth extraction, platelet-rich fibrin, osteogenesis, bone resorption, cone beam computed tomography

Posted Date: January 7th, 2022

DOI: https://doi.org/10.21203/rs.3.rs-1225393/v1

License: (9) This work is licensed under a Creative Commons Attribution 4.0 International License. Read Full License

Version of Record: A version of this preprint was published at BMC Oral Health on March 23rd, 2022. See the published version at https://doi.org/10.1186/s12903-022-02126-0. 


\section{Abstract}

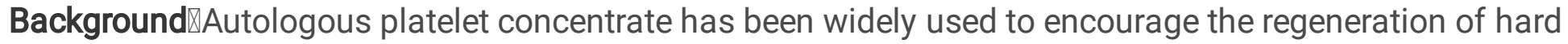
and soft tissues. Up to now, there are three generations of autologous platelet concentrates. Many studies have shown that different autologous platelet concentrates have different healing effects. However, these differences still need to be further verified and discussed. The purpose of this study was to explore and compare the effects of platelet-rich fibrin, concentrated growth factor and platelet-poor plasma on the healing of tooth extraction sockets in New Zealand rabbits.

Methods $\ A$ total of 24 healthy male New Zealand white rabbits aged 8-12 weeks were selected. The experimental animals were randomly divided into four groups: three experimental groups were respectively implanted with PPP, CGF and PRF gel after bilateral mandibular anterior teeth were extracted, and the control group did not implant any material. The alveolar bone of the mandibular anterior region was taken at 2, 4 and 8 weeks after operation. The height and width of the extraction wound were detected by CBCT, the growth of the new bone was observed by HE and Masson staining, and the expression of osteogenic genes was detected by real-time PCR. Data were analyzed using IBM SPSS statistical package 22.0 .

Results: The radiological results showed that alveolar bone absorption in all groups gradually increased over time. However, the experimental groups showed lower amounts of bone absorption. The histological results showed that new bone formation was observed in all groups. Over time, the new bone trabeculae of the CGF group became closely aligned while those in the PPP and PRF groups remained scattered. PCR results showed that the expression of BMP-2 and ALP was higher in the experimental groups than the control group.

Conclusion: In conclusion, the application of PRF, CGF and PPP in tooth extraction sockets effectively promoted bone regeneration. CGF showed more effective bone induction and tissue regeneration ability in the long term.

\section{Background}

Alveolar bone after tooth extraction undergoes bone resorption due to lack of dental support and functional stimulation, while bone reconstruction is accompanied by osteoclast resorption and fibrous bone filling [1]. $70 \%-80 \%$ of the bone loss occurs in the first 3 months after tooth extraction [2].

The resorption and atrophy of alveolar bone will have an adverse effect on subsequent restoration treatment, especially implant treatment. They increase the difficulty of implant operation and are not conducive to the stability of implants. Therefore, methods of alveolar sockets preservation are worthy of further exploration, so as to reduce the absorption of the alveolar ridge at the tooth extraction site, and provide more adequate bone volume and a favorable alveolar ridge shape for subsequent treatment. Numerous studies have been dedicated to evaluating the efficacy of different socket-filling biomaterials. Autogenous and allogeneic bone grafts have been recognized as frequently-used methods for decades, 
however, several limitations, such as extra site of surgery and prolonged surgery, an uncertain infection rate and limited autologous bone alternatives, have restricted their widespread development [3]. The use of synthetic biomaterials as alternative products has continued to develop subsequently, especially prior to implantation [4-5]. But most exogenous biomaterials still have some uncertainties in bone mineral binding ability, biodegradability and effective antibacterial ability [6].

In recent years, autologous plasma products, such as platelet-rich fibrin (PRF), concentrated growth factor (CGF) and platelet-poor plasma (PPP) have attracted the attention of researchers due to their ability to promote new bone formation [7] and tissue regeneration [8]. PRF is a second-generation platelet concentrate product which is easy to produce without any biological agents. Many studies have suggested that the use of PRF can promote bone regeneration through the release of cytokines after activation [9-10], but others have shown that there is a lack of standardization in its production and application, and that small differences may lead to variable clinical effects [11]. CGF was introduced by Sacco in 2006 [12]. Unlike PRF, it is centrifuged using special centrifuges, and different centrifugation speeds result in a larger, denser, and more abundant growth factor fibrin matrix [13]. A large number of studies have confirmed the advantages of CGF in bone defect repair [14-15].

PPP is the supernatant of plasma after centrifugation that contains few platelets. Studies [16] showed that PPP seems to have the ability to facilitate wound healing-associated cell function. In recent years, some in vitro studies have compared the similar effects of PPP and PRP in innervation and muscle repair [17-18], but there are few studies evaluating the role of PPP in bone tissue, and the results are divergent. Hamdan et al [19] at the cellular level have shown that the difference of concentration due to the vitro test will lead to a great difference in the results, which is also an important factor limiting the development of PPP.

Alveolar bone is the most active bone tissue in the body. Compared with other parts of bone defects, there will be significant absorption in the tooth extraction without intervention, which puts forward high requirements for the effect of bone induction materials used for tooth extraction. At present, some studies on the application of autologous plasma products in the tooth extraction have no consistent or robust results, and most of their studies are limited with the combined application of other osteogenic induction materials, and there are few independent studies on the effect of plasma products without additives. Further carefully designed and long-term observation cycles and multiple observation levels are needed to explore their respective strengths. Therefore, the aim of this study is to compare the effects of PRF, CGF and PPP on the healing of tooth extraction sockets in rabbits and to compare their long-term effects and influence characteristics of different stages from 2 weeks, 4 weeks and 8 weeks continuous observation. And in order to preliminary explore the mechanism of concrete, we also compare their influence on osteogenesis related genes, aims to provide guidance for clinical choice medicine.

\section{Materials And Methods}

\subsection{Sample size calculation}


The sample size was calculated using PASS 15.0 software (NCSS, LLC, Utah, USA). The statistical design was based on comparisons of the bone absorption rate between the PRF, CGF, PPP and control groups. The analysis module One-Way-Analysis of Variance F-Tests in the Means was used. According to the results of the preliminary experiment, the mean bone absorption rate in the PPP, CGF, PRF and control groups was $14 \%, 13 \%, 16 \%$, and $39 \%$ respectively. The standard deviation was set as $12 \%$. Statistical significance was set as $a=0.05$, with four groups, statistical power of 0.9 , and a group allocation ratio of 1: 1: 1. With these parameters, the sample size needed for the current study was six in each group (Fig. 1).

\subsection{Animals and study design}

All of the research protocols used in study were approved by the ethical committee of Southwest Medical University, Luzhou, China (Certificate number 201906-1). A randomized controlled study was conducted according to the ARRIVE guidelines [20]. Healthy male New Zealand White rabbits weighing $2.0-2.5 \mathrm{~kg}$ (average $2.2 \mathrm{~kg}$ ) and aged 8-12 weeks each were used in this study. All animals were purchased from the Department of Animal Science Central of Southwest Medical University and were taken good care of by professional laboratory technicians. They were housed in a temperature $\left(22 \pm 2^{\circ} \mathrm{C}\right)$ and humidity $(55 \pm 5 \%)$ controlled room under a 12/12 h light/dark cycle and kept in separate cages, with free access to food and water. After 2 weeks of observation, the experimental treatment was carried out.

\subsection{Preparation of autologous PPP, CGF, and PRF}

$9 \mathrm{ml}$ venous blood from the ear veins of each rabbit were drawn and collected into sterile vacuum tubes without additive (Greiner BioOne, Kremsmünster). The samples were immediately put into a Medifuge MF200 (Silfradent srl, Forlì, Italy), and centrifugation was carried out according to a preset procedure: acceleration for 30 seconds, then 2 minutes at $2700 \mathrm{rpm}, 4$ minutes at $2400 \mathrm{rpm}, 4$ minutes at $2700 \mathrm{rpm}$, 3 minutes at $3000 \mathrm{rpm}$, deceleration for 36 seconds, and stop [13]. This process separated the samples into three layers: a red blood cell (RBC) layer that covered the lower part of the tube, a CGF layer that covered the middle part and a PPP layer that covered the upper part (Fig. 2). The PPP was activated for experimental use with $10 \%$ calcium chloride. The CGF and activated PPP gel were thus collected for experimental use.

Based on a previously described protocol [21], $9 \mathrm{ml}$ venous blood was collected into a centrifuge tube without any anticoagulant. After centrifuging immediately for $10 \mathrm{~min}$ at 3,000 rpm, the whole blood separated into two layers, the lower layer being the RBC layer, and the upper layer being the PRF layer (Fig. 2).

\subsection{Surgical procedure}


The animals were randomly and evenly divided into four groups. Three groups received PPP, CGF and PRF gel respectively, while the remaining control group did not receive any implant material. They all received an intramuscular injection of penicillin $(800,000$ units three times daily) for 3 days postoperatively. Intravenous injection of $30 \mathrm{mg} / \mathrm{kg}$ sodium pentobarbital (Sigma, St. Louis, MO, USA) through the ear margin was used for general anesthesia. After the anesthetic had taken effect, the gingiva was separated with a periosteal elevator, then the teeth were loosened with the elevator, and after that the bilateral mandibular anterior teeth were extracted. All the above procedures were performed by the same oral and maxillofacial surgeon. Subsequently, the materials were severally implanted into the tooth extraction sockets in the experimental groups (Fig. 3). After that, the extraction sockets were carefully sutured and closely observed for avoiding infection. Thereafter, at 2, 4 and 8 weeks after tooth extraction, three rabbits were randomly selected from each group and euthanized with an overdose of pentobarbital sodium. The bilateral mandible was taken as the specimen for subsequent analysis.

\subsection{Radiographic analysis}

All animals were scanned twice by cone beam computed tomography (CBCT I and CBCT II), once after tooth extraction surgery, and the other after euthanasia, when the alveolar bone around the mandibular anterior teeth was immediately removed for scanning. All sectional images were obtained by the same radiologist using a Kodak 9500CBCT scanner (Carestream Health, Rochester, NY, USA), with the following settings: exposure at $5.0 \mathrm{~mA}$ and $120 \mathrm{kV}$ for $9.6 \mathrm{~s}$ and axial slice thickness $0.2 \mathrm{~mm}$. The results were processed and analyzed by the same radiologist (who was blinded to the group allocation) using image analysis software (CS Imaging Version 7.0.23.0.d, Carestream Health, Rochester, NY, USA). Changes in alveolar bone width $(\mathrm{ABW})$ and alveolar bone height $(\mathrm{ABH})$ were observed. Three sections were selected for each CBCT to measure the height and width respectively, and each section was randomly measured three times. ABW was measured using the method of Chen et al [22]. Measurements were performed on cross-sectional slices in the apical, median, and coronal third of the socket. $\mathrm{ABH}$ was measured using a method described previously by Liu et al [23]. Measurement was carried out on three sagittal planes, namely the buccal plane of the extraction socket, the lingual plane, and the middle plane of the first two planes. The changes in $\mathrm{ABW}$ and $\mathrm{ABH}$ were expressed by the measured value of $\mathrm{CBCT}$ before tooth extraction (CBCT I) minus the measured value of CBCT after euthanasia (CBCT II).

\subsection{Histological analysis}

After fixing in 10\% paraformaldehyde solution for $48 \mathrm{~h}$, the samples were demineralized in 10\% EDTA solution (North Tianyi Chemical Reagent Co. Ltd., Tianjin, China) for 5 weeks, washed, dehydrated, and paraffin embedded (Paraplast; Kendall Healthcare, Mansfield, MA, USA), parallel to the long axis of the tooth, comprising a continuous section in the buccal and lingual direction with a section thickness of 5 $\mu \mathrm{m}$, and then stained with hematoxylin and eosin(H\&E). Masson's trichrome stain was carried out in the same way. The above processes were performed by a histological technician who was blinded to the 
experimental protocols. An optical microscope (Olympus BX43, Olympus Corporation, Tokyo, Japan) with a magnification of $\times 200$ was used for observation, and a digital camera installed on the microscope was used to obtain images.

\subsection{Real-time quantitative polymerase chain reaction (RT- qPCR)}

Real-time quantitative polymerase chain reaction (RT-qPCR) was used to detect the expression of two markers of osteogenic genes: a differentiation marker of early osteoblasts - alkaline phosphatase (ALP), and a differentiation marker of late osteoblasts - bone morphogenetic protein-2 (BMP-2). After sacrificing the rabbits, bone tissue was obtained from the tooth extraction sockets and immediately stored in liquid nitrogen and ground in a mortar. A chloroform-free RNA extraction kit (BioTeke, Beijing, China) was used to extract total RNA from the samples. Then, according to the manufacturer's instructions, $1 \mu \mathrm{g}$ of RNA was reverse transcribed into cDNA, using ReverTra Ace qPCR RT Master Mix (TOYOBO, Japan), and stored at $-20^{\circ} \mathrm{C}$ before use. The CDNA was used as the template for real-time quantitative polymerase chain reaction (RT-qPCR). The total volume of the amplification reaction system was $20 \mu \mathrm{l}$, including $6 \mu \mathrm{l}$ primers, $12 \mu \mathrm{l}$ of QuantiNova STBR Green PCR (Qiagen, Germany), $1.5 \mu \mathrm{l}$ cDNA and $2.5 \mu \mathrm{lddH_{2 }}$ O. The primers were purchased from Sangon Biotech Co. (Shanghai, China). The sequences were: 5'-TCCCACTTTGTCTGGAACCG-3' and 5'-TCCTGTTCAGCTCGTACTGC-3' for ALP, 5'AGGAAGCTTTGGGAGACGAC-3' and 5'-AAGTGGGTCACTTCCACCAC-3' for BMP, and 5'GTGGCATCCTGACGCTCAAGTAC-3' and 5'-AAGCTCGTTGTAGAAGGTGTGGTG-3' for $\beta$-actin.

\subsection{Statistical analysis}

Data were analyzed using IBM SPSS statistical package 22.0 (IBM Co., Chicago, USA). Categorical variables are presented as the mean \pm standard deviation (SD). One-way analysis of variance (ANOVA) with the Student-Newman-Keuls (SNK) comparison test was employed to detect differences among different groups. A significance level of 0.05 was chosen.

\section{Results}

\subsection{Clinical results}

No accidental deaths occurred. All animals were in good physical condition and had a good diet. No infection or other complications occurred in any of the tooth extraction sockets. after surgery. On the 5th day after operation, the tooth extraction wound surface was completely covered by epithelial tissue.

\subsection{Radiological analysis}


The $A B W$ and $A B H$ gradually increased in all groups over time. However, the experimental groups showed lower amounts of bone absorption.

Two weeks after surgery, the $A B W$ and $A B H$ in each group revealed different degrees of absorption (Table 1). Both $A B W$ and $A B H$ indicated a significantly lower rate of absorption in the PPP group than in the other three groups $(P<0.05)$. The $A B W$ and $A B H$ at 4 and 8 weeks postoperatively are shown in Table 2 and Table 3. In short, the CGF group showed the lowest absorption, followed by the PPP and PRF groups, with the highest absorption in the control group. There were no significant differences in ABW between the PRF group and the control group at 4 weeks $(P>0.05)$, or in ABH between the PPP group and CGF group at 8 weeks $(P>0.05)$.

\subsection{Histological analysis}

Two weeks after surgery, new bone formation was observed in the tooth extraction sockets in the PPP group, with the new bone extending from the lateral wall to the center. There were abundant osteoblasts and active proliferation. Osteoblasts were arranged in rows around the bone matrix, and some bone trabeculae and mature fibrous tissue could be seen. The new bone trabeculae were thinner in the CGF group and PRF group than in the PPP group, and the arrangement was irregular, with blood vessels growing into the extraction sockets in the PRF group. A large number of inflammatory cells and a small number of fibroblasts were found in the sockets in the control group, and there were few new bones and bone trabeculae.

By the 4th week, each of the experimental groups had formed a larger amount of new bone than the control group. In the CGF group, the new bone further increased and continued to extend towards the center of the sockets, the osteoblasts proliferated actively, the bone trabeculae were more abundant the new bones were connected with each other, and the fibrous connective tissue and inflammatory cells had decreased. New bone formation in the sockets of the PPP group also increased significantly, but there were slightly fewer osteoblasts than in the CGF group, and the bone trabeculae were thinner. The growth of new bone in the PRF group was weaker than that in the PPP group, but there was more neovascularization. In the control group, the small amount of new bone tissue was scattered.

By the 8th week, new bone had formed in the extraction alveolus in the CGF group, the new bone trabeculae were closely connected and arranged similarly to the normal state, and the trabeculae were thick and calcified, but there was still a small amount of fibrous connective tissue. The new bone in the extraction sockets was thinner in the PPP group followed by the PFR group than that in the CGF group, but the bone tissue of the medial wall of the tooth extraction fossa was more mature and partially fused with the surrounding bone tissue. New bone formation could be seen in the control group, but it was significantly less extensive than in the experimental group, and osteoblasts and blood vessel density were relatively rare (Fig. 4 and Fig. 5). 


\subsection{RT-qPCR analysis}

Expression of alkaline phosphatase (ALP): The expression of ALP in the PPP group was the highest at 2 weeks after surgery $(P<0.05)$. At 4 weeks, ALP expression was significantly higher in the CGF group than in the other three groups. By the 8th week, there was no significant difference in ALP activity among the four groups (Fig. 6).

Expression of bone morphogenetic protein-2 (BMP-2): BMP expression was significantly higher in the PRF group than in the other groups at 2 weeks. The PRF group still had the highest expression at 4 weeks, and at the same time, the expression of BMP in the PPP group and CGF group was gradually increasing. By the 8th week, with the development of bone remodeling, BMP expression was the highest in the CGF group, followed by the PRF group and PPP group, which all showed higher expression than the control group $(P<0.05)$ (Fig. 7).

\section{Discussion}

A series of events occur during the healing of tooth extraction sockets, including (1) blood clot formation, (2) fibroblast infiltration and vascular endothelial cell proliferation, (3) connective tissue hyperplasia, (4) fibrous bone formation, and (5) mature bone tissue establishment. The healing process involves interaction between various cells and growth factors [24]. Therefore, improving the microenvironment of the tooth extraction sockets can promote healing [25] and induce bone tissue regeneration.

PRF and CGF are platelet concentrates containing a large number of growth factors [26], including transforming growth factor (TGF- $\beta$ ), platelet-derived growth factor (PDGF), vascular endothelial growth factor (VEGF), and basic fibroblast growth factor (BFGF). These growth factors participate in events such as osteoblastic movement, proliferation and differentiation [27], so as to regulate the activity of osteoblasts and promote bone regeneration.

PPP is the upper component of autologous plasma products. Compared with PRF and CGF, it contains fewer platelets, but is rich in plasma growth factor and fibrinogen. Chen et al [28] showed that fibrinogen may have a more significant effect on bone regeneration, suggesting that PPP has the potential to promote bone regeneration in the early stages of healing. In this study, PPP, CGF and PRF were used in extraction sockets separately to determine their effect on alveolar bone healing and resorption, with a view to developing new clinical approaches.

The radiographic and histological analyses revealed large areas of new alveolar bone in all three experimental groups at all time points, except for the control group, where only a small amount of new alveolar bone was generated, similar to the findings of other scholars. For example, Hatakeyama et al [29] found that in their vivo study of rat calvarial bone defects, the bone defects were almost filled with bone tissue 8 weeks after surgery and treatment with CGF and PPP gel, while a few control (untreated) defects were still apparent at 8 weeks. Similarly, Kim et al [30] observed that in their comparative study on rabbit- 
skull defect healing, the PRP, PRF and CGF groups had all formed a higher amount of new bone than the control group by the 6th week after surgery.

Overall, CGF and PPP were slightly more effective than PRF at promoting extraction socket healing during the time points observed in our study. The reason for this may be that the fibrin network of insoluble fibrin provides a scaffold for the cells and serves as a substrate for the continuous release of growth factors, and the cells are exposed to fibrin molecules exhibiting three-dimensional cell-cell interactions [31], allowing the growth factors to continuously act on the extraction sockets, promoting osteoblast proliferation and differentiation as well as reducing the absorption of the alveolar bone. Moreover, Hatakeyama et al [29] showed that significantly more fibrinogen was contained within PPP than in PRF, and Isobe et al [32] found that CGF gels contained thicker fibrin fibers than PRF gels based on scanning electron microscopic examination. Both findings support our results. In addition, both the PRF and CGF groups displayed fewer functions at 2 weeks. This may be because CGF and PRF, which contain a large number of platelets, are not only reservoirs of growth factors but also immune nodes containing a large number of inflammatory mediators. Inflammatory factors such as a -granules released after their activation may limit the differentiation of osteoblast-related cells during early healing process [33-34].

During the growth of osteoblasts, the expression of specific genes varies at different stages [35]. The peak expression of genes reflects the developmental sequence of osteocyte differentiation, which can be divided into three main stages: proliferation, extracellular matrix maturation and mineralization. Some scholars have proposed that the regulation of genes in this developmental sequence depends on the maturation of osteoblasts [36]. ALP activity is closely related to bone growth and remodeling, and its expression can reflect the early differentiation of osteoblasts and the maturity of bone tissue [37]. BMP-2 promotes the maturation and function of osteoblasts and bone remodeling by inducing the differentiation of mesenchymal bone progenitor cells [38]. It is a representative osteogenic product in the late stage of osteogenic differentiation. Our results showed that ALP was highly expressed in early osteogenesis and BMP was highly expressed in late osteogenesis, which was similar to other scholars' studies. Lu et al. showed high levels of expression of ALP in the early stage of osteogenesis, but with the development of bone maturation and matrix mineralization, it reduced gradually, and the late marker of osteoblast differentiation increased [39]. In addition, our results suggested that the PPP group expressed a high level of ALP in 2 weeks, but CGF group reach the high level significantly in the 4 weeks, the reasons for this phenomenon may be credit to their different biological structures. CGF owns dense fiber structure and slow release of growth factors [40].

One limitation of the study is that it failed to further explore the molecular biological mechanisms underlying the effect of PRF, CGF and PPP on the healing of tooth extraction sockets. However, real time PCR were preliminarily used to observed the expression level of osteogenic genes in different groups. In addition, platelet concentrates could be used in combination with other material to optimize their effects. Further clinical study would be conducted to verify and supplement the results of this study.

\section{Conclusions}


PPP, PRF and CGF can promote the healing of tooth extraction sockets, promote new bone formation, reduce bone resorption, and improve the expression of osteogenesis-related genes. Yet considering their long-term effects, CGF shows greater benefits in osteogenesis, resulting in efficient bone induction and tissue regeneration. Since the components of PPP, CGF and PRF are all derived from autologous blood without immunogenicity, their preparation is simple. Furthermore, they have good biocompatibility and appropriate biodegradability when they are implanted into the tooth extraction sockets.

\section{Declarations}

\section{Funding}

The authors sincerely appreciate the financial support from National Training Program of Innovation and Entrepreneurship for Undergraduates of China (S202010632035, S202010632002), Office of Science \& Technology and Talent Work of Luzhou (2021-JYJ-67) and Southwest Medical University (2021ZKZD007).

\section{Author Contributions}

The first two authors (Siying Li and Hongyi Yang) contributed equally to this work and they are co-first authors. Siying Li, Hongyi Yang and Yun He contributed to the conception and design of the study. Siying Li, Hongyi Yang, Qinyu Duan, Hongyu Bao, Aodi Li, and Wei Li performed the acquisition of the data (laboratory or clinical) and the statistical analysis. Siying Li, Hongyi Yang wrote the first draft of the manuscript. Siying Li, Hongyi Yang and Yun He approved the final version of manuscript. All authors approved the submitted version.

\section{Acknowledgements}

Not applicable

\section{Competing interests}

The authors declare that they have no competing interests.

\section{Ethics approval}

The study was performed in accordance with the guidelines of the ARRIVE guidelines(2010).All of the research protocols used in study were approved by the ethical committee of Southwest Medical University, Luzhou, China (Certificate number 201906-1). 


\section{Availability of data and materials}

The datasets generated and/or analysed during the current study are not publicly available due to the ongoing related further research projects but are available from the corresponding author on reasonable request.

\section{Consent for publication}

Not applicable.

\section{References}

1. Barone A, Ricci M, Tonelli P, Santini S, Covani U. Tissue changes of extraction sockets in humans: a comparison of spontaneous healing vs. ridge preservation with secondary soft tissue healing. Clin Oral Implants Res. 2013;24:1231-1237.

2. Discepoli N, Vignoletti F, Laino L, de Sanctis M, Muñoz F, Sanz M.. Early healing of the alveolar process after tooth extraction: an experimental study in the beagle dog. J Clin Periodontol. 2013;40:638-644.

3. Stumbras A, Kuliesius P, Januzis G, Juodzbalys G. Alveolar Ridge Preservation after Tooth Extraction Using Different Bone Graft Materials and Autologous Platelet Concentrates: a Systematic Review. J Oral Maxillofac Res. 2019;10:e2.

4. Gholami GA, Najafi B, Mashhadiabbas F, Goetz W, Najafi S. Clinical, histologic and histomorphometric evaluation of socket preservation using a synthetic nanocrystalline hydroxyapatite in comparison with a bovine xenograft: a randomized clinical trial. Clin Oral Implants Res. 2012;23:1198-1204.

5. Adel-Khattab D, Afifi NS, Abu El Sadat SM, Aboul-Fotouh MN, Tarek K, Horowitz RA. Bone regeneration and graft material resorption in extraction sockets grafted with bioactive silica-calcium phosphate composite (SCPC) versus non-grafted sockets: clinical, radiographic, and histological findings. J Periodontal Implant Sci. 2020;50:418-434.

6. Chen ZY, Gao S, Zhang YW, Zhou RB, Zhou F. Antibacterial biomaterials in bone tissue engineering. $J$ Mater Chem B. 2021;9:2594-2612.

7. Zhang Z, Li X, Zhao J, Jia W, Wang Z.. Effect of autogenous growth factors released from platelet concentrates on the osteogenic differentiation of periodontal ligament fibroblasts: a comparative study. PeerJ. 2019; 7:e7984.

8. Kim TH, Kim SH, Sándor GK, Kim YD. Comparison of platelet-rich plasma (PRP), platelet-rich fibrin (PRF), and concentrated growth factor (CGF) in rabbit-skull defect healing. Arch Oral Biol. 2014; 59:550-558.

9. Li, F., Jiang, P., Pan, J., Liu, C., \& Zheng, L. Synergistic application of platelet-rich fibrin and $1 \%$ alendronate in periodontal bone regeneration: A meta-analysis. BioMed research international, 2019. 
10. Castro AB, Meschi N, Temmerman A, Pinto N., Lambrechts P, Teughels W, et al. Regenerative potential of leucocyte- and platelet-rich fibrin. Part A: intra-bony defects, furcation defects and periodontal plastic surgery. A systematic review and meta-analysis. J Clin Periodontol. 2017;44:67-82.

11. Fernández-Medina T, Vaquette C, Ivanovski S. Systematic Comparison of the Effect of Four ClinicalGrade Platelet Rich Hemoderivatives on Osteoblast Behaviour. Int J Mol Sci. 2019; 20(24):6243.

12. Sacco L. International Academy of Implant Prosthesis and Osteoconnection. In: Lecture. 2006.

13. Rodella LF, Favero G, Boninsegna R, Buffoli B, Labanca M, Scarì G, et al. Growth factors, CD34 positive cells, and fibrin network analysis in concentrated growth factors fraction. Microsc Res Tech. 2011;74:772-777.

14. Talaat WM, Ghoneim MM, Salah O, Adly OA. Autologous Bone Marrow Concentrates and Concentrated Growth Factors Accelerate Bone Regeneration After Enucleation of Mandibular Pathologic Lesions. J Craniofac Surg. 2018;29:992-997.

15. Xu Y, Qiu J, Sun Q, Yan S, Wang W, Yang P, et al. One-Year Results Evaluating the Effects of Concentrated Growth Factors on the Healing of Intrabony Defects Treated with or without Bone Substitute in Chronic Periodontitis. Med Sci Monit. 2019;25:4384-4389.

16. Creeper F, Lichanska AM, Marshall RI, Seymour GJ, Ivanovski S. The effect of platelet-rich plasma on osteoblast and periodontal ligament cell migration, proliferation and differentiation. J Periodontal Res. 2009;44:258-265.

17. Martínez CE, González SA, Palma V, Smith PC. Platelet-Poor and Platelet-Rich Plasma Stimulate Bone Lineage Differentiation in Periodontal Ligament Stem Cells. J Periodontol. 2016;87:e18-e26.

18. Song D, Huang Y, Van Dessel J, Shujaat S, Orhan K, Vangansewinkel T, et al. Effect of platelet-rich and platelet-poor plasma on peri-implant innervation in dog mandibles. Int J Implant Dent. 2019;5:40.

19. Chellini F, Tani A, Zecchi-Orlandini S, Sassoli C. Influence of Platelet-Rich and Platelet-Poor Plasma on Endogenous Mechanisms of Skeletal Muscle Repair/Regeneration. Int J Mol Sci. 2019;20:683.

20. Hamdan AA, Loty S, Isaac J, Bouchard P, Berdal A, Sautier JM. Platelet-poor plasma stimulates the proliferation but inhibits the differentiation of rat osteoblastic cells in vitro. Clin Oral Implants Res. Jun. 2009;20(6):616-23.

21. Kilkenny C, Browne W, Cuthill IC, Emerson M, Altman DG; NC3Rs Reporting Guidelines Working Group. Animal research: reporting in vivo experiments: the ARRIVE guidelines. Br J Pharmacol. 2010;160:1577-1579.

22. Dohan DM, Choukroun J, Diss A, Dohan SL, Dohan AJ, Mouhyi J, Gogly B. Platelet-rich fibrin (PRF): a second-generation platelet concentrate. Part I: technological concepts and evolution. Oral Surg Oral Med Oral Pathol Oral Radiol Endod. 2006;101:e37-e44.

23. Chen J, He Y, Shan C, Pan Q, Li M, Xia D. Topical combined application of dexamethasone, vitamin C, and $\beta$-sodium glycerophosphate for healing the extraction socket in rabbits. Int J Oral Maxillofac Surg. 2015;44(10):1317-1323. 
24. Liu X, Zhang X, Tian X, Chen J, He Y. Effect of osteogenic inducer sustained release system on bone remodeling after tooth extraction. Acts Universitatis Medicinalis Anhui. 2021;56(5):823-827.

25. Smane L, Pilmane M. Evaluation of the presence of MMP-2, TIMP-2, BMP2/4, and TGF 33 in the facial tissue of children with cleft lip and palate. Acta Med Litu. 2018;25:86-94.

26. Wang X, Ma J, Wang Z, Xiao L. The clinical efficacy of using autologous platelet-rich plasma in total hip arthroplasty: A retrospective comparative study. Medicine (Baltimore). 2018;97:e12451.

27. Lee HM, Shen EC, Shen JT, Fu E, Chiu HC, Hsia YJ. Tensile strength, growth factor content and proliferation activities for two platelet concentrates of platelet-rich fibrin and concentrated growth factor. J Dent Sci. 2020;15:141-146.

28. Li H, Sun S, Liu H, Chen H, Rong X, Lou J, et al. Use of a biological reactor and platelet-rich plasma for the construction of tissue-engineered bone to repair articular cartilage defects. Exp Ther Med. 2016;12:711-719.

29. Chen TL, Liang XJ, Zhang XH. Do the Fibrin Scaffold and Growth Factors in Platelet-Rich Fibrin Play the Most Vital Roles in Bone Regeneration? A Critical Comment. J Craniofac Surg.2019;30:19231926.

30. Hatakeyama I, Marukawa E, Takahashi Y, Omura K. Effects of platelet-poor plasma, platelet-rich plasma, and platelet-rich fibrin on healing of extraction sockets with buccal dehiscence in dogs. Tissue Eng Part A. 2014;20:874-882.

31. Kim TH, Kim SH, Sándor GK, Kim YD. Comparison of platelet-rich plasma (PRP), platelet-rich fibrin (PRF), and concentrated growth factor (CGF) in rabbit-skull defect healing. Arch Oral Biol. 2014;59:550-558.

32. Kawase T, Okuda K, Saito Y, Yoshie H. In vitro evidence that the biological effects of platelet-rich plasma on periodontal ligament cells is not mediated solely by constituent transforming-growth factor-beta or platelet-derived growth factor. J Periodontol.2005;76:760-767.

33. Isobe $\mathrm{K}$, Watanebe $\mathrm{T}$, Kawabata $\mathrm{H}$, Kitamura $\mathrm{Y}$, Okudera T, Okudera $\mathrm{H}$, et al.Mechanical and degradation properties of advanced platelet-rich fibrin (A-PRF), concentrated growth factors (CGF), and platelet-poor plasma-derived fibrin (PPTF). Int J Implant Dent.2017;3:17.

34. Hong S, Chen W, Jiang B. A Comparative Evaluation of Concentrated Growth Factor and Platelet-rich Fibrin on the Proliferation, Migration, and Differentiation of Human Stem Cells of the Apical Papilla. J Endod.2018;44:977-983.

35. Golebiewska EM, Poole AW. Platelet secretion: From haemostasis to wound healing and beyond. Blood Rev.2015;29:153-162.

36. Yoon JY, Kim TS, Ahn JH, Yoon JU, Kim HJ, Kim EJ. Remifentanil promotes osteoblastogenesis by upregulating Runx2/osterix expression in preosteoblastic C2C12 cells. J Dent Anesth Pain Med. 2019;19:91-99.

37. Lian J B, Stein G S. Concepts of osteoblast growth and differentiation: basis for modulation of bone cell development and tissue formation. Crit Rev Oral Biol Med. 1992;3: 269-305. 
38. Tang H, Kankala RK, Wang S, Chen A. Supercritical fluid-assisted controllable fabrication of open and highly interconnected porous scaffolds for bone tissue engineering. Sci China Life Sci. 2019;62:1670-1682.

39. Mai Z, Peng Z, Wu S, Zhang J, Chen L, Liang H, et al. Single bout short duration fluid shear stress induces osteogenic differentiation of MC3T3-E1 cells via integrin $\beta 1$ and BMP2 signaling cross-talk. PLoS One. 2013;8(4):e61600.

40. Lu Y, Li L, Zhu Y, Wang X, Li M, Lin Z, et al. Multifunctional Copper-Containing Carboxymethyl Chitosan/Alginate Scaffolds for Eradicating Clinical Bacterial Infection and Promoting Bone Formation. ACS Appl Mater Interfaces. 2018;10(1):127-138.

41. Tian S, Wang J, Dong F, Du N, Li W, Song P, et al. Concentrated Growth Factor Promotes Dental Pulp Cells Proliferation and Mineralization and Facilitates Recovery of Dental Pulp Tissue. Med Sci Monit. 2019;25:10016-10028.

\section{Tables}

\section{TABLE 1}

Resorption of Alveolar Bone Height and Width at Week 2 According to Groups

\begin{tabular}{|c|c|c|}
\hline Group & Absorption of $\mathbf{A B W}(\mathrm{mm})$ & Absorption of $\mathbf{A B H}(\mathrm{mm})$ \\
\hline control & $0.21 \pm 0.03$ & $0.63 \pm 0.06$ \\
\hline PPP & $0.13 \pm 0.02^{a, d}$ & $0.44 \pm 0.03^{a, d}$ \\
\hline CGF & $0.18 \pm 0.04$ & $0.51 \pm 0.03^{\mathrm{a}}$ \\
\hline PRF & $0.20 \pm 0.03^{b}$ & $0.56 \pm 0.08^{b}$ \\
\hline$F$ & 4.000 & 13.544 \\
\hline$P$-value & $\nabla 0.05$ & $\nabla 0.05$ \\
\hline
\end{tabular}

ABW, alveolar bone width; ABH, alveolar bone height; PPP, Platelet-Poor Plasma;

CGF, Concentrated Growth Factor; PRF, Platelet-Rich Fibrin

Data represent mean \pm SD.

a Statistically significant difference compared to the control group ( $P \square 0.05$ ).

b Statistically significant difference compared to the PPP group ( $P \bowtie 0.05)$.

c Statistically significant difference compared to the CGF group ( $P \llbracket 0.05$ ).

d Statistically significant difference compared to the PRF group ( $P \square 0.05)$. 
TABLE 2

Resorption of Alveolar Bone Height and Width at Week 4 According to Groups

\begin{tabular}{|c|c|c|}
\hline Group & Absorption of $\mathbf{A B W}(\mathrm{mm})$ & Absorption of $\mathbf{A B H}(\mathrm{mm})$ \\
\hline control & $0.72 \pm 0.08$ & $1.21 \pm 0.06$ \\
\hline PPP & $0.55 \pm 0.06^{a}$ & $0.91 \pm 0.08^{a, c, d}$ \\
\hline CGF & $0.48 \pm 0.03^{a, d}$ & $0.76 \pm 0.08 \mathrm{a}, \mathrm{b}, \mathrm{d}$ \\
\hline PRF & $0.63 \pm 0.05^{c}$ & $1.10 \pm 0.05^{b, c}$ \\
\hline$F$ & 9.582 & 26.600 \\
\hline$P$-value & $\varangle 0.05$ & $₫ 0.05$ \\
\hline
\end{tabular}

ABW, alveolar bone width; $\mathrm{ABH}$, alveolar bone height; PPP, Platelet-Poor Plasma;

CGF, Concentrated Growth Factor; PRF, Platelet-Rich Fibrin

Data represent mean \pm SD.

a Statistically significant difference compared to the control group ( $P \square 0.05$ ).

b Statistically significant difference compared to the PPP group ( $P \bowtie 0.05)$.

c Statistically significant difference compared to the CGF group ( $P \square 0.05$ ).

d Statistically significant difference compared to the PRF group ( $P \square 0.05$ ).

TABLE 3

Resorption of Alveolar Bone Height and Width at Week 8 According to Groups 


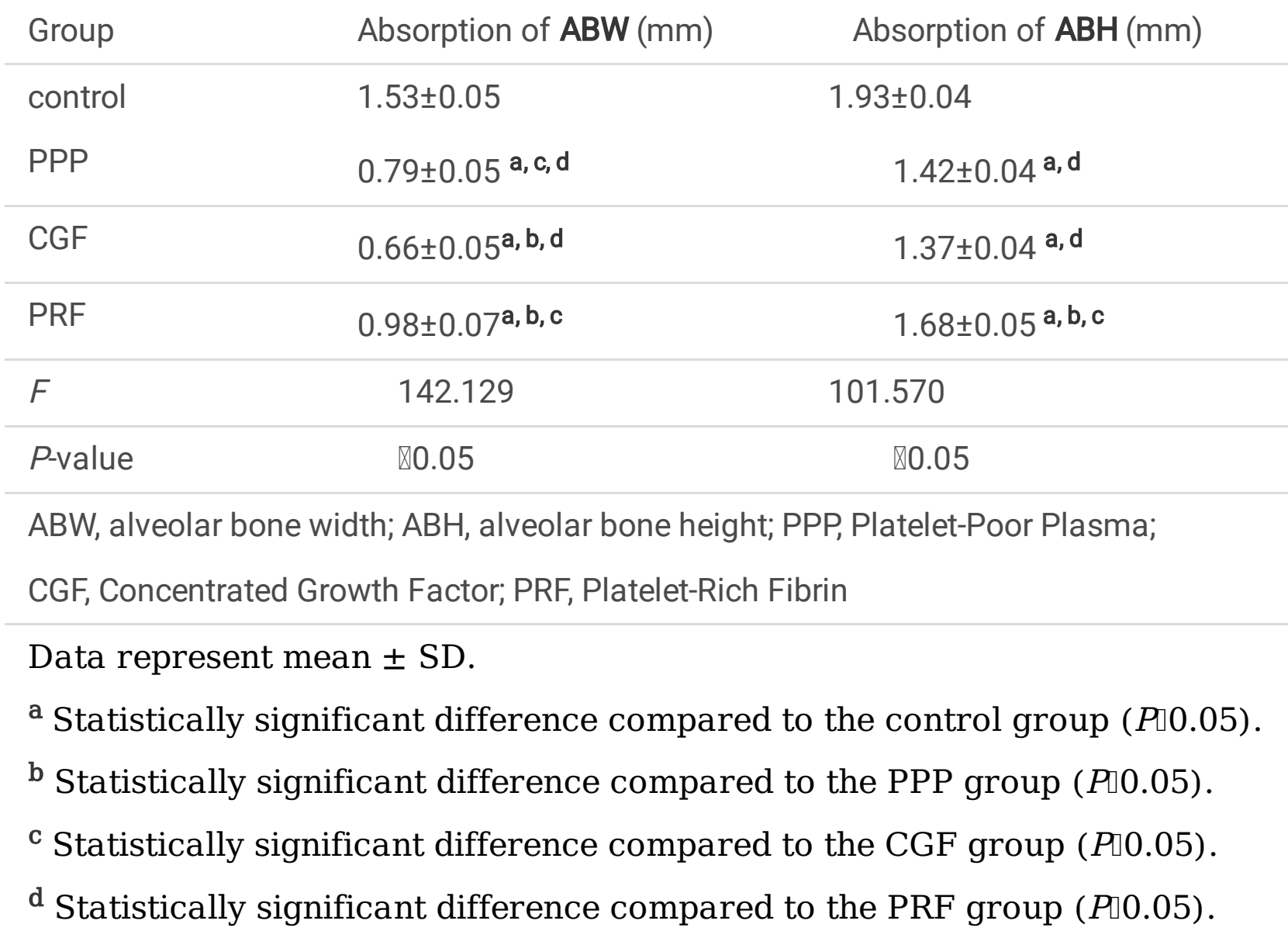

\section{Figures}

PASS 15.0.5

One-Way Analysis of Variance F-Tests

\section{Numeric Results}

Means: 16131439

\begin{tabular}{|c|c|c|c|c|c|c|c|c|}
\hline & Average & & Total & & $\begin{array}{r}\text { Std Dev } \\
\text { of Means }\end{array}$ & $\begin{array}{l}\text { Standard } \\
\text { Deviation }\end{array}$ & Effect & \\
\hline Power & $\mathbf{n}$ & G & $\mathbf{N}$ & K & $\sigma m$ & $\sigma$ & Size & Alpha \\
\hline 0.9581 & 5.00 & 4 & 20 & 1.00 & 10.74 & 10.00 & 1.0735 & 0.0500 \\
\hline 0.9298 & 6.00 & 4 & 24 & 1.00 & 10.74 & 12.00 & 0.8946 & 0.0500 \\
\hline 0.9367 & 7.00 & 4 & 28 & 1.00 & 10.74 & 13.00 & 0.8258 & 0.0500 \\
\hline 0.9013 & 8.00 & 4 & 32 & 1.00 & 10.74 & 15.00 & 0.7157 & 0.0500 \\
\hline
\end{tabular}

Figure 1

Sample size calculation process 

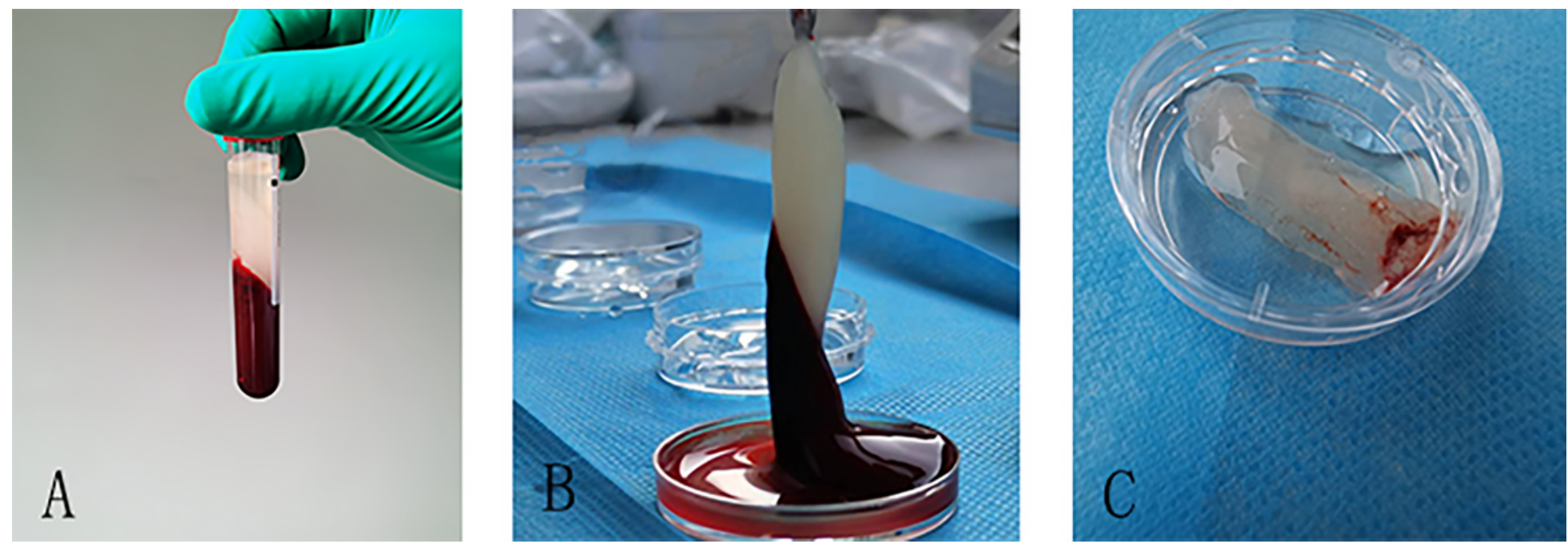

\section{Figure 2}

(A)After setting the CGF preparation program and rotating for 12 minutes, the test tube was divided into three layers.

(B)Separate the three layers.

(C)Store the CGF gel in sterile saline solution for later use.
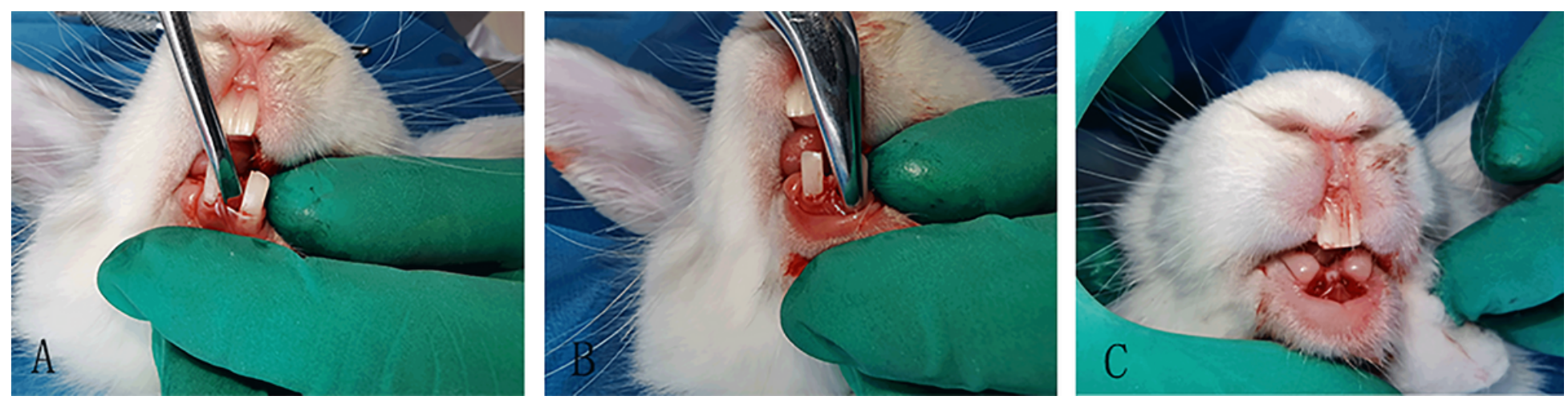

Figure 3

(A)Loosen the lower front teeth with appropriate force.

(B)Use the extractor to hold the tooth, shake and pull out.

(C)After the extraction of both lower anterior teeth, the extraction wounds were stitched. 


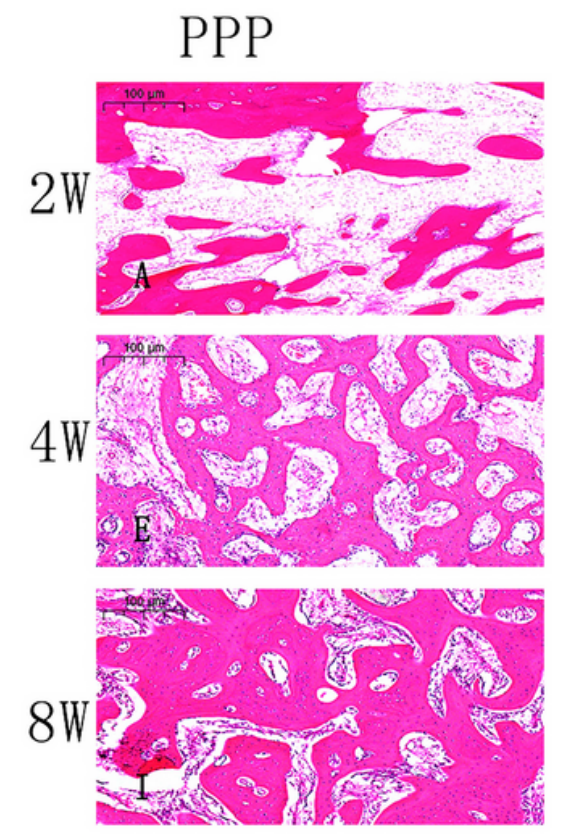

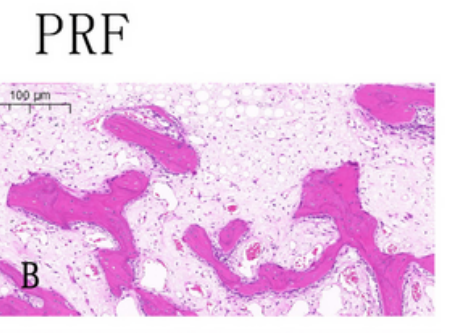

\section{CGF}
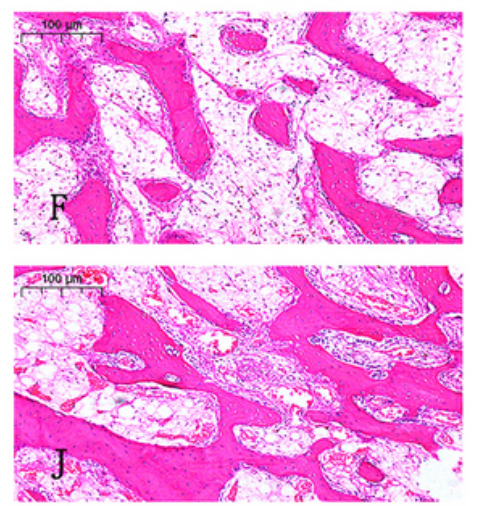

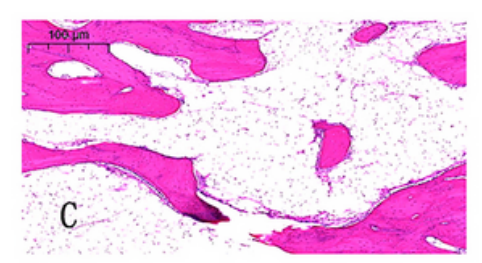

\section{Control}
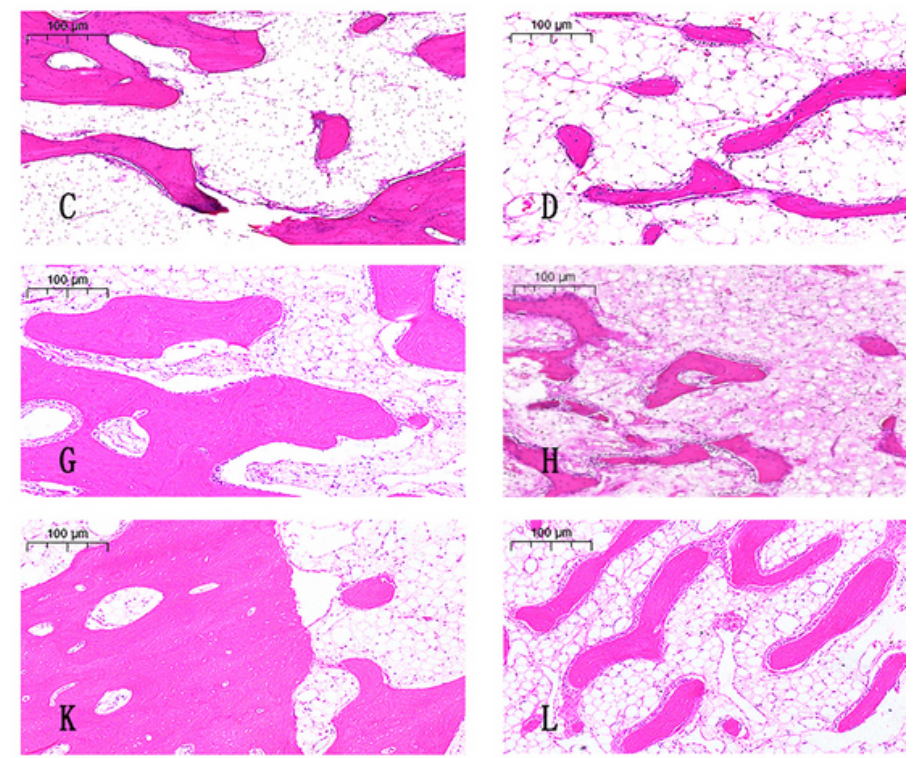

Figure 4

H\&E staining of the Tooth extraction at 2 weeks, 4 weeks and 8 weeks. Scar bar $=100 \mu m$
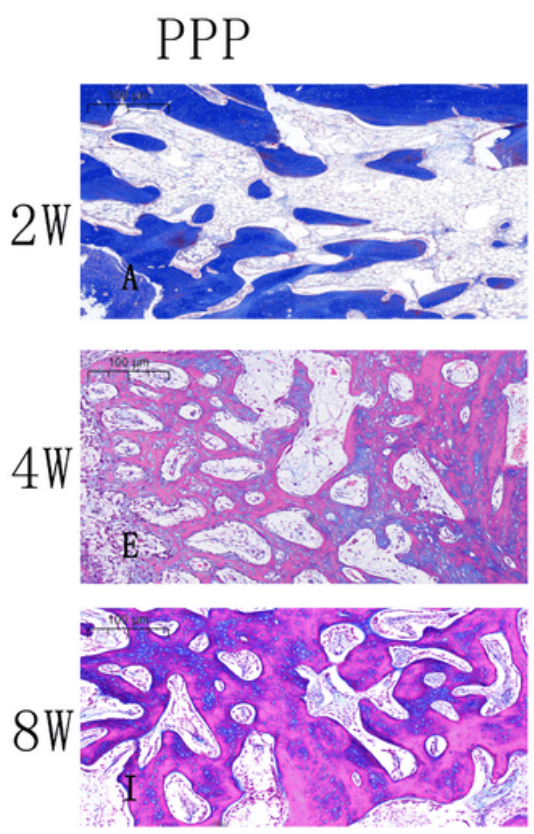

PRF
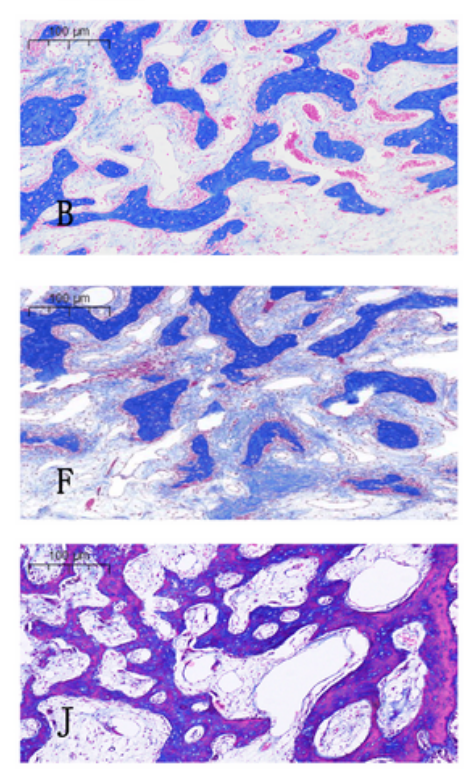

CGF
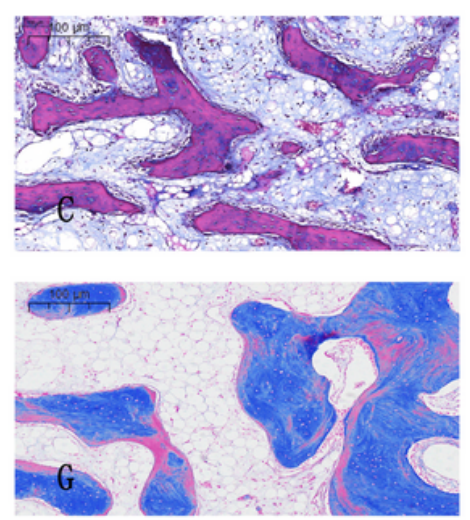

Control
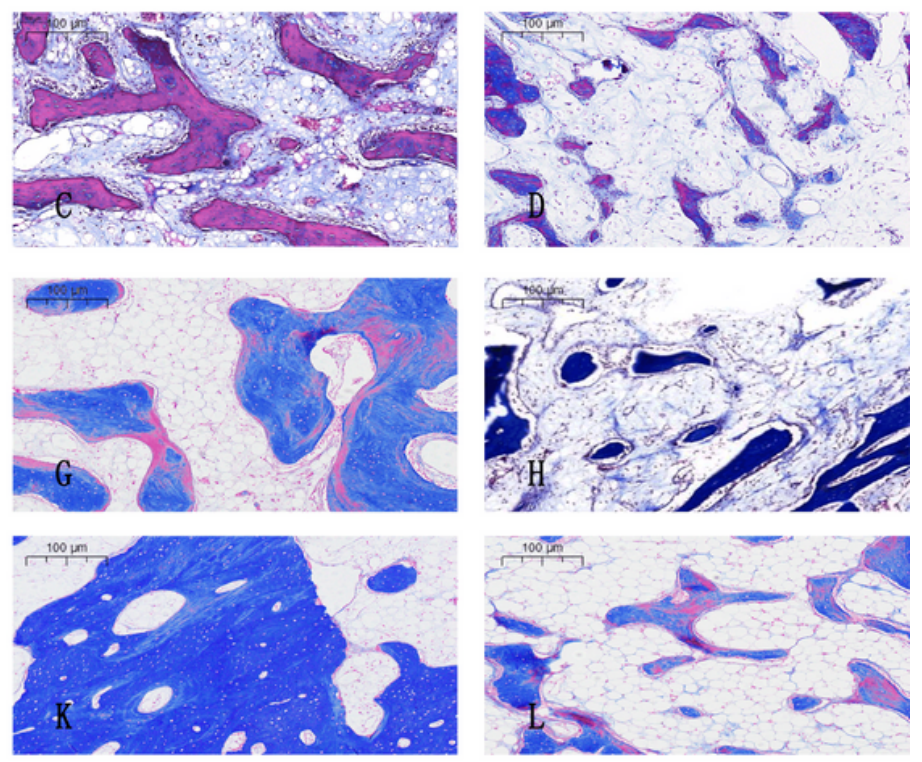

Figure 5

Masson staining of the Tooth extraction at 2 weeks, 4 weeks and 8 weeks. Scar bar $=100 \mu \mathrm{m}$ 

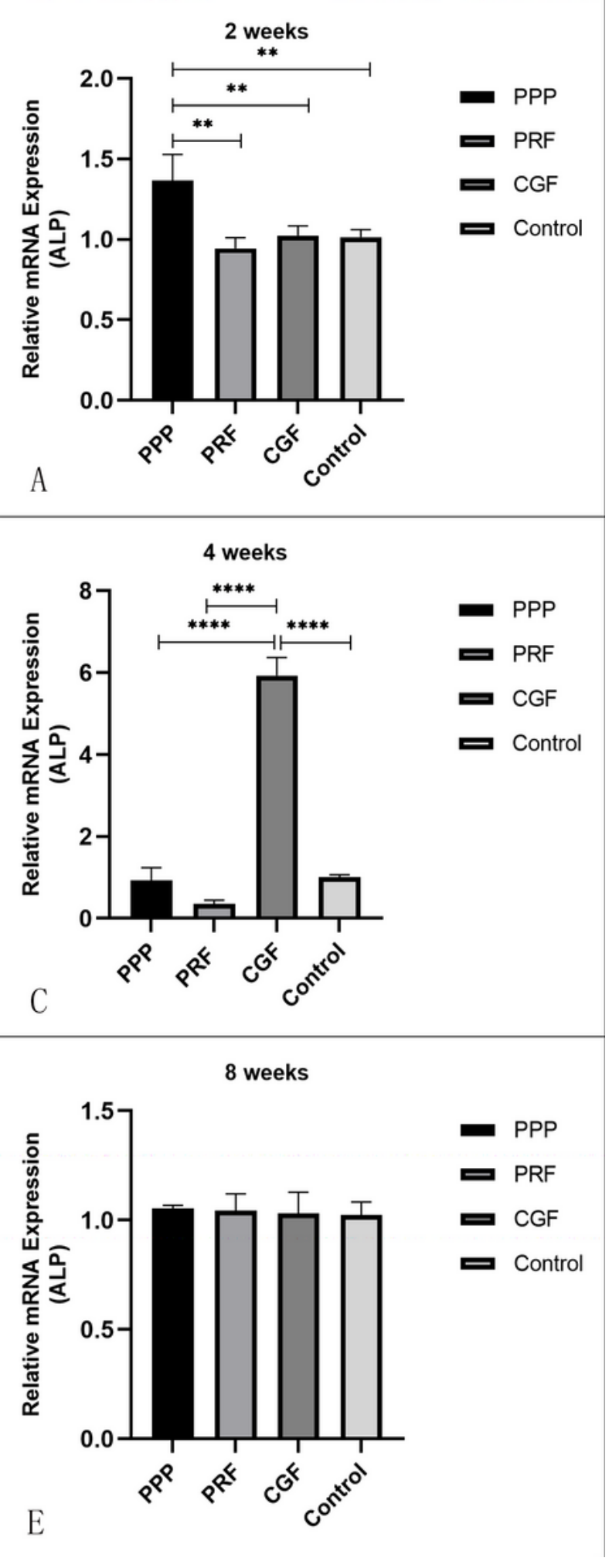

Figure 6

Relative mRNA expression levels of different marker genes $\triangle A L P \otimes i n$ bone tissue after tooth extraction filling with PPP, PRF, CGF and control group healed for 2 weeks, 4 weeks and 8 weeks. ** refers to $P$ $<0.01 \rrbracket^{\star \star \star \star}$ refers to $P<0.001 \rrbracket^{\star \star \star \star \star}$ refers to $P<0.0001$ 

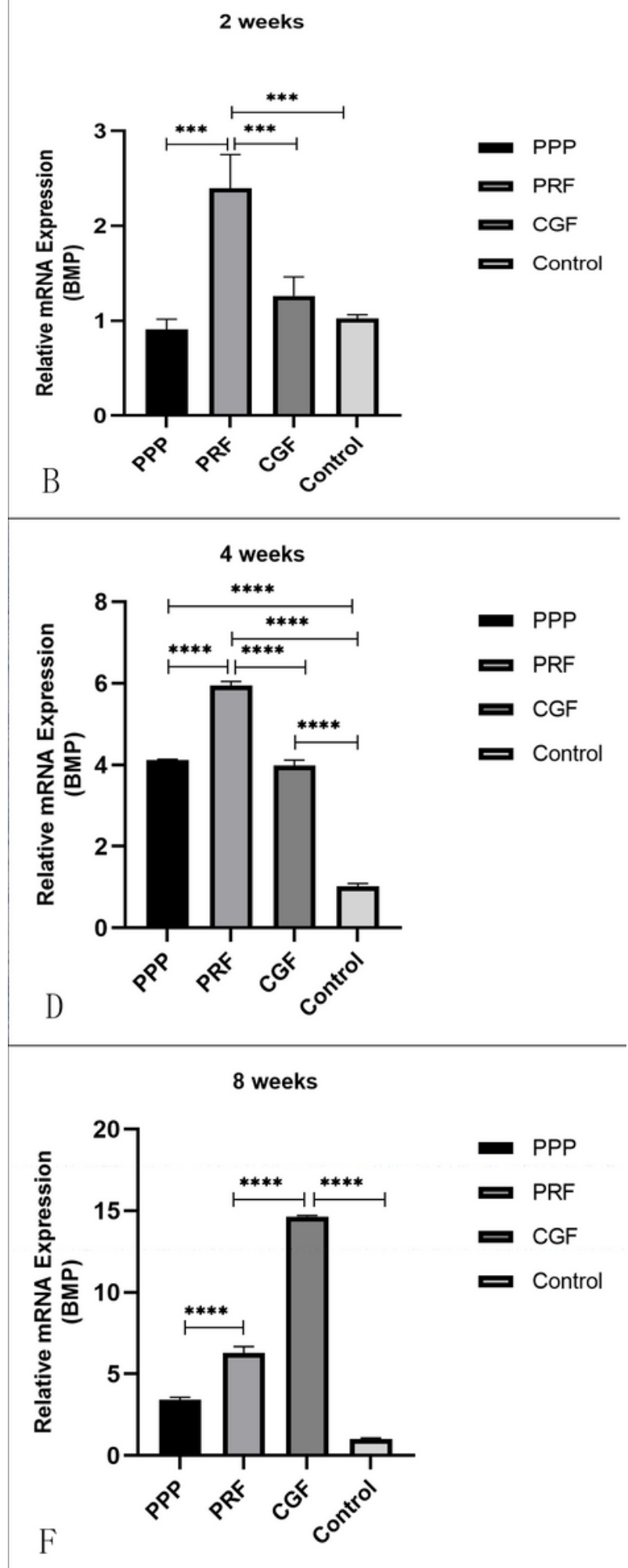

Figure 7

Relative mRNA expression levels of different marker genes $\triangle B M P \bigotimes$ in bone tissue after tooth extraction filling with PPP, PRF, CGF and control group healed for 2 weeks, 4 weeks and 8 weeks. ** refers to $P$ $<0.01 \nabla^{\star \star \star \star}$ refers to $P<0.001 \nabla^{\star \star \star \star \star}$ refers to $P<0.0001$ 